\title{
The Observation Scale for Autism (OSA): A New Screening Method to Detect Autism Spectrum Disorder before Age Three Years
}

\author{
Nils Haglund ${ }^{1, *}$, SvenOlof Dahlgren ${ }^{2}$, Karin Källén $^{3}$, Peik Gustafsson ${ }^{1}$ and Maria Råstam ${ }^{1}$ \\ ${ }^{1}$ Department of Clinical Sciences Lund, Child and Adolescent Psychiatry, Lund University, Sweden \\ ${ }^{2}$ Department of Psychology, University of Gothenburg, Sweden \\ ${ }^{3}$ Department of Clinical Sciences Lund, Obstetrics and Gynecology, Center of Reproduction Epidemiology, \\ University of Lund, Sweden
}

\begin{abstract}
Background: There is an increasing body of evidence that early interventions for children with Autism Spectrum Disorder (ASD) promote a positive development of social interaction. Thus, tools for early detection of ASD are warranted.

Aim: Development of, and deciding cut-off-levels for, a new screening tool for ASD, the Observation Scale for Autism (OSA). The OSA was designed to be used at the free health check-up at 30 months, offered to all children in Sweden.

Method: The OSA consists of 12 observations and takes less than 10 minutes to use. The performance of the test was investigated by assessing 37 children previously diagnosed ASD, 23 with Down Syndrome (DS) and 26 typically developing children (TD).

Results: Children diagnosed with ASD showed statistically significant higher scores in all 12 items compared to TD children, and significantly higher in 10 items compared to the children with DS. Most of the observations in OSA seemed to cover specific symptoms of ASD, but two of the observations were more related to developmental level. The nine most discriminative items for ASD were identified, and among those, a cut-off limit was chosen ( $\geq 3$ items). Among children with ASD, 34/37 reached the proposed cut off, compared to 0/26 and 4/23 among children in the TD and DS groups, respectively.
\end{abstract}

Conclusion: The results suggest that the OSA discriminates children with ASD from TD children and children with DS. Using the suggested cut off, OSA provides high sensitivity for ASD (92\%) with a very low false positive rate.

Keywords: Screening, Autism, sensitivity, Intellectual disability, typically developing children.

\section{INTRODUCTION}

Early detection of Autism Spectrum Disorders (ASD) has been an important field for research in autism, especially after the publication of studies showing that early intervention and treatment of ASD symptoms are associated with better outcome [1,2]. Different screening instruments have been developed for detection of ASD and there is a discussion of what would be the optimal age for finding ASD symptoms. Convergent data indicate that ASD symptoms emerge in the first two years of life [3-5]. In a recent review [6], it was concluded that both retrospective and prospective studies showed robust evidence that behavioral signs of ASD can be detected early in infancy.

In Sweden, all children are offered a free health check at 30 months of age. If there was a screening tool for ASD which was quick and easy to use for Child Health Center nurses, this would offer an opportunity to detect children with ASD in early childhood. Ideally, the

*Address correspondence to this author at the Dept. of Clinical Sciences, Lund, Child and Adolescent Psychiatry, Lund University, Barav. 1C, SE-221 85 Lund, Sweden; Tel: +46 40 6753669; E-mail: nils.haglund@med.lu.se

E-ISSN: 2292-2598/15 screening tool would be an objective instrument, not dependent on parent's propensities to recognize their children's developmental shortcomings. The results from such a screening could then be used to select children for referral to neuro-psychiatric units for detailed examinations to establish (or reject) a diagnosis of autism.

There are few scales available for the assessment of symptoms of ASD in children 3 years and younger. Most of them are based on parent-reported questionnaires, either solely [7-13], or designed to be used in combination with an observation instrument $[14,15]$. To our knowledge, there are only two published instruments that are entirely based on an observation scheme [16-17]. Both are designed to be used by trained autism nurses, and thus, not suitable for ASD screening performed by Child Health Center nurses.

We conclude that although some appropriate screening tools for early detection of ASD have been developed and shown to have good psychometric properties, there is a need for brief, easy to handle assessment instruments designed for use in the primary health care system. Furthermore, it has been 
reported that existing screening tools depending on parents' observation abilities have unsatisfactory value in discriminating between ASD and non-ASD within the group of children showing abnormal development [18].

The purpose was to develop a screening observation scale for ASD to be used in primary care 30-months follow-up, to examine the performance of the instrument on a small sample of children, and to decide the suitable ASD cut-off limit before testing the instrument on the population. Based on the small sample, we also attempted to evaluate if the OSA could discriminate children with ASD from TD children and from children with a general developmental delay. The latter group was represented by children with DS. We also attempted to identify the most ASD discriminatory observations (the most autism specific items).

\section{METHODS}

\section{Participants}

Three groups of children participated, children with ASD $(n=37)$, children with DS $(n=23)$ and TD children $(n=26)$ (Table 1).

The ASD group consisted of 31 children diagnosed with DSM-IV/ICD-10 autistic disorder/childhood autism and six children with DSM-IV/ICD-10 PDDNOS/atypical autism. They had all been assessed at age 28-36 months. During the diagnostic process all children in the ASD group had been evaluated with the Autism Diagnostic Observation Schedule [19] and the Autism Diagnostic Interview- Revised [20].

The children with DS were diagnosed at birth. Age at the assessment with the OSA was 31-55 months.
DS was used as a proxy for intellectual disability [21]. In the DS group, in order to minimize the risk to include children with DS and ASD combined, the parent and/or caretaker were asked before the assessment if they had any suspicion that the child had autistic traits. The children in the two clinical groups, ASD and DS, were assessed with the OSA by psychologists.

The TD children were all recruited from the same preschool. They were assessed at the age of $24-40$ months with the OSA by a preschool teacher.

\section{Measure}

The OSA was developed by the first two authors (SO.D, NH). The items in the OSA were chosen according to research on early markers in autism $[3,5,6]$. The OSA was developed to be used by trained $\mathrm{CHC}$-nurses. The focus is on the observation of the behaviour of the child and the child's ability to interact with his/her parent(s) and the CHC-nurse. In a second step, the OSA was adapted by the first two authors in collaboration with two CHC-units in Malmoe to be used at the standard 30-month assessment of all children, which is a part of the existing Child Health program in Sweden. The $\mathrm{CHC}$ units were chosen from two areas of the city that represent different cultural and language population background. The OSA was designed to be used as a part of a larger examination of the child and had to be easy to administer, to demand minimal formal training, and could not be time consuming.

The OSA consists of 12 observations, especially focused on reciprocal behavior in communication, social interaction and play, namely reciprocal social interaction between caregiver and child; reciprocal eye

Table 1: Background Characteristics by Study Group

\begin{tabular}{|c|c|c|c|c|c|c|}
\hline & \multicolumn{2}{|c|}{ ASD $n=37$} & \multicolumn{2}{|c|}{ DS $n=23$} & \multicolumn{2}{|c|}{ TD $n=26$} \\
\hline & $\mathbf{N}$ & (\%) & $\mathbf{n}$ & (\%) & $\mathbf{n}$ & (\%) \\
\hline Boys & 34 & (92) & 14 & (61) & 8 & (31) \\
\hline Girls & 3 & (8) & 9 & (39) & 18 & (69) \\
\hline \multicolumn{7}{|l|}{ Language group } \\
\hline Swedish & 27 & $(73,0)$ & 15 & $(65,2)$ & 23 & $(88,5)$ \\
\hline Other Nordic languages & 1 & $(2,7)$ & 0 & (0) & 0 & $(0)$ \\
\hline Other Western European languages & 0 & (0) & 0 & (0) & 1 & $(3,8)$ \\
\hline Eastern European languages & 1 & $(2,7)$ & 1 & $(4,3)$ & 1 & $(3,8)$ \\
\hline Arabic & 8 & $(21,6)$ & 7 & $(30,4)$ & 0 & $(0)$ \\
\hline African, Sub-Saharan languages & 0 & (0) & 0 & (0) & 0 & $(0)$ \\
\hline Asian languages & 0 & (0) & 0 & (0) & 1 & $(3,8)$ \\
\hline
\end{tabular}

ASD; Autism Spectrum Disorders, DS; Down Syndrome, TD; Typically Developing children. 
contact between nurse and child during the assessment; reciprocal play between nurse and child; the child's spontaneous use of two word phrases during assessment. The instruction for the observer was to determine whether the child behaved at each observation point as expected for a child with the developmental age of 30 months. If not, the observer would score one point.

\section{Statistical Analysis}

Pair-wise group comparisons (ASD vs TD, and ASD vs DS), respectively, were performed using Fisher's exact tests. The comparisons regarding the age at assessment across groups was made by one-wayANOVA with Bonferroni post-hoc test. The comparison in gender representation, OSA scores and the with-in group analyses were made with Fisher's exact tests. The area under the Receiver Operating Characteristic (ROC) curve was used to estimate the overall ability of the OSA to detect autism among children at 2.5 years of age. In this analysis, children with DS were excluded. Statistical analyses were made using SPSS version 22.

\section{Ethics}

The study was approved by the Regional Ethics Committee in Lund (Dnr 2010/366, Dnr 2011/299). Parents in all three groups were given oral and written information and gave consent for participation of their child. All data were anonymised before being forwarded to the research team.

\section{RESULTS}

The basic characteristics of the three study groups are displayed in Table 1. In the ASD group there was a majority of boys participating (92\%), and so was the case in the group of children with DS (64\%). In the group of TD children there were a majority of girls $(69 \%)$ (difference in gender distribution, Chi2(2) $=25.4$, $p<.001)$. There were statistically significant differences across groups concerning age at assessment $(F=$ 8.392, $\mathrm{df}=2, \mathrm{p}<.001$ ). The mean age at assessment was 34.4 months in the ASD group, 39.7 months in the DS group, and 32.0 months in the group of TD children ( $p$ for difference ASD vs TD, $p=.49$; ASD vs DS, $p=0.12$; and $D S$ vs TD, $p<.001$ ). The majority of all participating children had Swedish as native language (ASD group $73 \%$; DS group $65 \%$; TD group $89 \%$ (difference across groups, Chi2(2)=3.81, $\mathrm{p}=.15$ ). The second most common language in the ASD and DS groups was the Arabic language (Table 1).

Most of the children in the ASD group (92\%) had more than 4 scores on the 12-item OSA version compared to $17 \%$ in the DS group, and $0 \%$ in the TD group. In the ASD group $46 \%$ of the children scored 8 or more on the OSA while none in the DS group scored more than 6 (Figure 1).

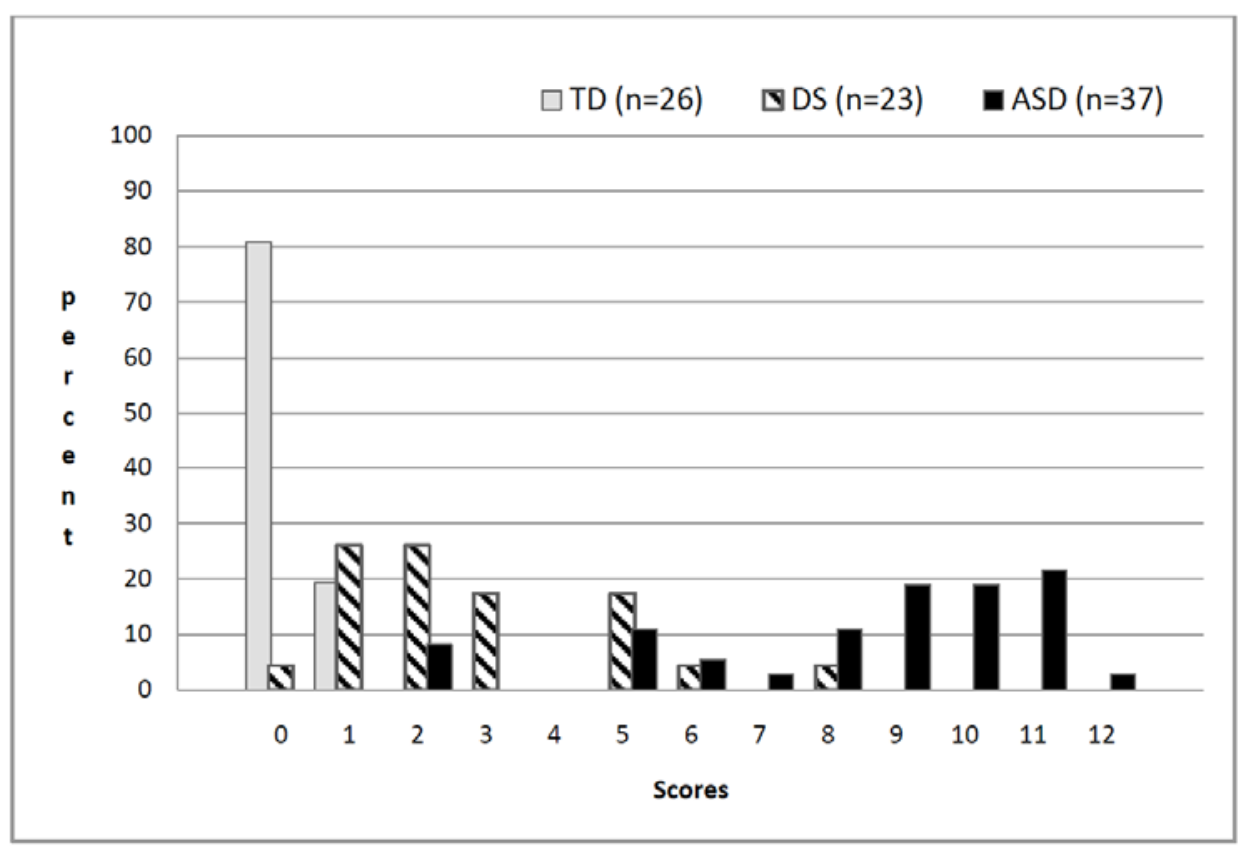

Figure 1: Number of scores by study group.

TD: Typically Developing, DS: Down Syndrome, ASD: Autism Spectrum Disorders. 
Table 2: Number of Scores by Task and Study Group. The p-Values were Obtained by Fisher Exact Tests

\begin{tabular}{|c|c|c|c|c|c|c|c|c|}
\hline \multirow[b]{2}{*}{ Items } & \multicolumn{2}{|c|}{ ASD $n=37$} & \multicolumn{2}{|c|}{ DS $n=23$} & \multicolumn{2}{|c|}{ TD $n=26$} & \multirow{2}{*}{$\begin{array}{c}\text { ASD vs DS } \\
p \text {-value }\end{array}$} & \multirow{2}{*}{$\frac{\text { ASD vs TD }}{p \text {-value }}$} \\
\hline & n scores & $(\%)$ & n scores & $(\%)$ & n scores & $(\%)$ & & \\
\hline Name recognition & 22 & 59,5 & 1 & 4,3 & 0 & 0 & $<.001$ & $<.001$ \\
\hline $\begin{array}{l}\text { Adequate response to } \\
\text { removal of toy }\end{array}$ & 29 & 78,4 & 4 & 17,4 & 1 & 3,8 & $<.001$ & $<.001$ \\
\hline Adequate movements & 23 & 62,2 & 14 & 60,9 & 2 & 7,7 & 1.0 & $<.001$ \\
\hline Interplay with parents & 31 & 83,8 & 6 & 26,1 & 0 & 0 & $<.001$ & $<.001$ \\
\hline Adequate eye contact & 15 & 40,5 & 0 & 0 & 0 & 0 & $<.001$ & $<.001$ \\
\hline Following pointing direction & 27 & 73 & 3 & 13 & 0 & 0 & $<.001$ & $<.001$ \\
\hline Pretend play & 31 & 83,8 & 2 & 8,7 & 0 & 0 & $<.001$ & $<.001$ \\
\hline Child pointing & 34 & 91,9 & 9 & 39,1 & 0 & 0 & $<.001$ & $<.001$ \\
\hline Building blocks & 18 & 48,6 & 4 & 17,4 & 0 & 0 & .026 & $<.001$ \\
\hline Kicking ball & 26 & 70,3 & 1 & 4,3 & 0 & 0 & $<.001$ & $<.001$ \\
\hline Two-word-sentences & 29 & 78,4 & 18 & 78,3 & 2 & 7,7 & 1.0 & $<.001$ \\
\hline Waves good-bye & 25 & 67,6 & 2 & 8,7 & 0 & 0 & $<.001$ & $<.001$ \\
\hline
\end{tabular}

ASD; Autism Spectrum Disorders, DS; Down Syndrome, TD; Typically Developing children.

Overall the children with ASD had statistically significant higher scores on all observations than the typically developing children (Table 2). Compared to the children with DS the children with ASD had statistically significant higher scores on ten of the twelve observations. The only two observations that did not reach statistically significance were "adequate movements" and "two word sentences" ( $p=1.0$ for both items).

The least frequent observations scored in the group with ASD were for "adequate eye contact" and "building blocks". The most frequent item scored were "child pointing", "pretend play", "interaction with parents", "two word sentences" and "adequate response to removal of toy" (Table 2). In the group with DS, observations related to developmental age were most frequently scored, i.e. "adequate movements" and "two word sentences". The only observations where typically developing children scored were "adequate movements", "two word sentences" and "adequate response to removal of toy".

In order to find out if the specific OSA observations were independent of native language, comparisons were made within the study groups. No significant differences were found. In the ASD group, the p-values for difference between language groups for the 12 items varied between .12 and 1.0. The corresponding $p$-values in the DS and TD groups ranged from $p=.13$ to 1.0 , and $p=1.0$, respectively (because of the low numbers of scores among children in the TD group only three items could be analysed). Concerning gender, there were no significant differences across groups ( $p$-value ranges: ASD $p=.12$ to 1.0 ; DS $p=.13$ to 1.0 ; TD $p=.30$ to .53 (only three observations analysed due to no variation).

In order to increase specificity, the nine most discriminative observations were selected (the 12 original, excluding the observations regarding adequate movements, building blocks, and two-word-sentences).

Table 3 shows the number of scores that each child in the respective study group obtained. Among the 37 children in the ASD group, 34 (92\%) scored for three observations or more The corresponding percentage among children in the DS and TD groups were 17\% $(3 / 23)$ and $0 \%(0 / 26)$, respectively. Thus, using three scores as a cut-off, the sensitivity for the OSA would be $92 \%$, and the false positive rate (1-specificity) among TD children would be $0 \%$. The corresponding area under the ROC-curve, showing the over-all ability of OSA to detect autism, was $0.998(95 \% \mathrm{Cl}$ : $0.994-$ 1.000).

Sub-analyses were performed, restricted to children who were between 28 and 40 months at assessment $(n=31, n=10, n=20$ for the ASD, DS, and TD groups, respectively). Within this sub-cohort, there was no agedistribution difference between the study groups $(F=$ 0.361, df $=2, p=.699$ ). In the ASD group, 29/31 children (94\%) scored for three observations or more. The corresponding percentage among children in the 
Table 3: Number of Scores among the 9 Most Discriminatory Variables ${ }^{A}$, by Study Group

\begin{tabular}{|c|c|c|c|c|c|c|c|c|c|c|c|}
\hline \multirow{4}{*}{$\begin{array}{l}\text { Number (i) } \\
\text { of scores }\end{array}$} & \multicolumn{9}{|c|}{ Study group } & \multicolumn{2}{|c|}{ Comparisons $^{B}$} \\
\hline & \multicolumn{3}{|c|}{ ASD $n=37$} & \multicolumn{3}{|c|}{ DS $n=23$} & \multicolumn{3}{|c|}{ TD $n=26$} & \multirow{3}{*}{$\begin{array}{c}\text { ASD vs DS } \\
p \text {-value }\end{array}$} & \multirow{3}{*}{$\begin{array}{c}\text { ASD vs } \\
\text { DS } \\
\text { p-value }\end{array}$} \\
\hline & \multirow{2}{*}{$\begin{array}{c}\text { Children } \\
\text { with (i) } \\
\text { scores } \\
n\end{array}$} & \multicolumn{2}{|c|}{$\begin{array}{l}\text { Children with } \\
\text { (i) scores or } \\
\text { more }\end{array}$} & \multirow{2}{*}{$\begin{array}{c}\text { Children with } \\
\text { (i) scores } \\
\mathbf{n}\end{array}$} & \multicolumn{2}{|c|}{$\begin{array}{l}\text { Children with } \\
\text { (i) scores or } \\
\text { more }\end{array}$} & \multirow{2}{*}{$\begin{array}{c}\text { Children } \\
\text { with (i) } \\
\text { scores } \\
n\end{array}$} & \multicolumn{2}{|c|}{$\begin{array}{c}\text { Children with } \\
\text { (i) scores or } \\
\text { more }\end{array}$} & & \\
\hline & & $\mathbf{n}$ & $(\%)$ & & $\mathbf{N}$ & $(\%)$ & & & $(\%)$ & & \\
\hline 0 & 0 & 37 & 100 & 9 & 23 & 100 & 25 & 25 & 100 & - & - \\
\hline 1 & 3 & 37 & 100 & 7 & 14 & 61 & 1 & 1 & 4 & $<.001$ & $<.001$ \\
\hline 2 & 0 & 34 & 92 & 3 & 7 & 30 & 0 & 0 & 0 & $<.001$ & $<.001$ \\
\hline 3 & 2 & 34 & 92 & 3 & 4 & 17 & 0 & 0 & 0 & $<.001$ & $<.001$ \\
\hline 4 & 3 & 32 & 86 & 0 & 1 & 4 & 0 & 0 & 0 & $<.001$ & $<.001$ \\
\hline 5 & 2 & 29 & 78 & 0 & 1 & 4 & 0 & 0 & 0 & $<.001$ & $<.001$ \\
\hline 6 & 4 & 25 & 73 & 1 & 1 & 4 & 0 & 0 & 0 & $<.001$ & $<.001$ \\
\hline 7 & 6 & 19 & 62 & 0 & 0 & 0 & 0 & 0 & 0 & $<.001$ & $<.001$ \\
\hline 8 & 10 & 9 & 46 & 0 & 0 & 0 & 0 & 0 & 0 & $<.001$ & $<.001$ \\
\hline 9 & 7 & 7 & 19 & 0 & 0 & 0 & 0 & 0 & 0 & 0.04 & 0.04 \\
\hline
\end{tabular}

ASD; Autism Spectrum Disorders, DS; Down Syndrome, TD; Typically Developing children.

${ }^{A}$ Kicking ball, pretend play, adequate response to removal of toy, waves goodbye, name recognition, following pointing direction, child pointing, interplay with parents, adequate eye contact.

${ }^{B}$ Number of children with at least (i) scores versus children with less than (i) scores.

DS and TD groups were $30 \%(3 / 10)$ and $0 \%(0 / 20)$, respectively, both significantly different from the rate in the ASD group ( $p<.001$ for both comparisons).

\section{DISCUSSION}

The OSA was designed for the detection of suspected ASD symptoms in children under the age three years. The scale was specially developed to minimize the influence of different culture and language background, and to be used within the primary child health care program to select children who would benefit from referral to child neuro-psychiatric centers. The children with ASD scored statistically significant higher on ten out of the twelve observation items compared with children with DS, and on all twelve observations compared to the TD group. Using the 9 most discriminative observations, with a cut off of $\geq 3$ scores, the sensitivity in the ASD group reached $92 \%$, without any false positive test results among TD children. Using the proposed cut off, the false positive rate among children with DS was also low (17\%). Although the sample was small, the results indicate that OSA managed to discriminate children with ASD from both TD children and children with $D S(p<.001$ for a positive test result, ASD group vs TD group, and ASD group vs DS group, respectively). Three children in the ASD group did not reach the proposed cut-off score.
Two of these children had originally been diagnosed with PDD-NOS and had few symptoms of ASD according to $t A D I-R$ and ADOS in the diagnostic assessment. The other 5 children diagnosed with PDDNOS did not, according to our assessment with OSA, differ from children diagnosed with autistic disorder regarding to total score.

The nine most discriminatory observations were all illustrating skills of social reciprocity. The two observations that did not reach statistical significance between children with ASD and children with DS were both depending on general developmental level ("two word sentences" and "adequate movements"). The two observations that were most frequent scored in the TD group were among the youngest children in the group (24-30 months). These observations were also related to general development ("adequate movements" and "two word sentences" i.e. the same most frequent observations as in the group of children with DS). These findings taken together implies that the nine most discriminative observation situations "kicking ball", "pretend play", "adequate response to removal of toy", "waves goodbye", "name recognition", "follow pointing direction", "child pointing", "interplay with parents" and "adequate eye contact" illustrates ASD symptoms or at least early signs of ASD. These findings are also consistent with findings from Barbaro \& Dissanayake [22], evaluating ASD markers at 24 months of age. 
They also found "follow simple commands" and "loss of skills" to be important markers for suspected ASD. To "follow simple commands" is indirectly included in the OSA. In the situation where the children point at different objects they do it on instructions from the observer. However "loss of skills" cannot be observed during the assessment and is therefore not included in the OSA. The children with ASD in the current study had proportionally low score in "eye contact" (40.5\%), compared to Barbaro \& Dissanayake [22], who found $86 \%$ of the children with ASD had deficits in eye contact. This behavior is known to be a core symptom and a key marker for ASD in the literature of autism $[23,24]$. However, even if the children with ASD in the current study had a proportionally low score for "eye contact", they still had a statistically significant higher score than the other two study groups, and "eye contact" was also among the nine most discriminatory observations.

The least discriminatory variables in our study were "building blocks", "inadequate movements", "two word sentences", all variables related to developmental level. Accordingly, these observations did not discriminate children with ASD from children with DS (with the exception of building blocks, $p=.026$ ), but from group of TD children. This finding is in line with Mitchell et al. [25], who found that early markers including social, communicative and motor deficits distinguished ASD from children with developmental delay. Developmental delay included children with developmental language delay, children with Down syndrome, children with intellectual disability as well as children with other forms of broad delay without any specific diagnostic label. They also found that early signs of ASD overlapped with other types of developmental delay which complicate differential diagnosis at an early age. This could also be found in the present study were children with DS did score on the nine most discriminatory observations even if it was at a low frequency. Since children with ASD scored statistically significant on all items compared to the typically developing children, and on ten of the items compared to the children with Down syndrome, it seems that the OSA contains suitable items to detect children with ASD.

It was also found that the assessment with OSA did not differ depending on gender or native language, the last not least important since it was constructed to bridge language and cultural differences. Cultural differences regarding the parental awareness of traits of autism in their offspring was indicated in a study by
Haglund \& Källén [26] in which children of Swedish origin were much more likely to be diagnosed with Asperger syndrome than were children of immigrants.

In a review, Norris \& Lecavalier [27] examined five care-giver completed rating scales for screening for ASD. The scales reviewed were: the Social Communication Questionnaire (SCQ) [9], Gilliam Autism Rating Scale/Gilliam Autism Rating ScaleSecond Edition (GARS/GARS-2) [14], Social Responsiveness Scale (SRS) [11], Autism Spectrum Screening Questionnaire (ASSQ) [28], and Asperger Syndrome Diagnostic Scale (ASDS) [29]. Only one of these, the SCQ [9], performed well in screening for ASD at age 3 years and older. This review indicated that caregiver- completed rating scales needs much more scientific research to give diagnostic validity.

One of the strengths of the OSA is that the instrument is not depending on the subjective observations by the caregivers, but by trained primary care nurses who perform health check-ups on hundreds of children each year. The OSA should not be mistaken for AOSI (Autism Observation Scale for Infants, 18-items observational measure) [17], or the STAT (Screening Tool for Autism in Toddlers \& Young Children, 12-item observations) [1], which both are similar to OSA with the important difference that they are designed to be used by trained autism nurses.

\section{LIMITATIONS}

One obvious limitation in our study was in the recruitment of the comparison groups. The children in the three study groups were not ideally matched for gender and age. However, since the results remained virtually unchanged when the analyses were restricted to children with similar age, the different age distributions among the study groups do not seem to have introduced any major bias. Another putative major limitation was the fact that the assessments of the participants in the DS group and the ASD group were conducted by psychologists, but by a preschool teacher in the TD group. Even though the preschool teacher had received a short introduction course in autism and OSA, it could not be precluded that the interpretation of the OSA test might differ between the evaluators. Also, the test was not blinded as all examiners were aware of the children's diagnosis (or no diagnosis). Furthermore, we did not have permission to perform more than one test for each child. Thus, it has not been possible to perform any test - retest- or inter observer agreement analyses. Despite these limitations, the results suggest 
that the OSA could be useful in screening for ASD in small children.

Children with DS have a special syndrome that includes cognitive and learning disabilities. This group was used as a proxy for children diagnosed with intellectual disability. Individuals with DS are known to have a general cognitive delay and to be intellectually disabled to some degree [30]. The identification of ASD in children with DS is complicated since the pattern of test scores across subscales among children with DS is not fully explored [31]. One of our aims was to explore if the OSA could discriminate autism from general developmental delay. Children diagnosed with intellectual disability at the age of 2.5 years almost always suffer from multiple disabilities, including autism. In the absence of a suitable control group diagnosed with developmental delay, we chose a group which we know have cognitive impairment, usually without autistic traits. We are, however, aware that children with DS do not represent the total population of children diagnosed with intellectual disabilities.

The results imply that OSA can be used with children with different cultural and language background as well as independent of gender. However, the statistical analyses were performed on very small groups so the results must be regarded with caution.

\section{CLINICAL IMPLICATIONS}

It is essential to develop screening instruments for the early detection of ASD to make sufficient support, services and treatment available for children with ASD and their families. It is of uttermost importance to note that the proposed screening tool is not intended to be a stand-alone instrument. The OSA could select children suitable for referral to child neuropsychiatric clinics, but the final diagnoses of autism should only be made at diagnostic units using more thorough diagnostic tools, such as e.g. ADOS and/or ADI. The OSA is designed to be a powerful first link in a care chain, aiming at an as early as possible diagnosis of autism so that interventions could be initiated without delay. An ongoing screening study will use the OSA for all 30months old children coming for their standard check-up at the $\mathrm{CHCs}$ in Malmoe to further examine the ability of this screening tool to successfully detect ASD traits in children of all cultural and language background.

\section{FUNDING}

This research was supported by ALF Foundation, Region Skåne and the Lindhaga Foundation.

\section{CONFLICT OF INTEREST STATEMENT}

All five authors have reported no conflict of interest.

\section{REFERENCES}

[1] Rogers SJ, Vismara LA. Evidence-based comprehensive treatments for early autism. J Clin Child Adolesc Psychol 2008; 37: 8-38. http://dx.doi.org/10.1080/15374410701817808

[2] Dawson G, Rogers S, Munson J, Smith M, Winter J, Greenson J, et al. Randomized controlled trial of an intervention for toddlers with autism: The Early Start Denver Model. Pediatrics 2010; 125: e17-e23. http://dx.doi.org/10.1542/peds.2009-0958

[3] Dahlgren SO, Gillberg C. Symptoms in the first two years of life. A preliminary population study of infantile autism. Eur Arch Psychiatry Neurol Sci 1989; 238(3): 169-74. http://dx.doi.org/10.1007/BF00451006

[4] Young R, Brewer N, Pattison C. Parental identification of early behavioral abnormalities in children with autistic disorder. Autism 2003; 7: 125-143.

http://dx.doi.org/10.1177/1362361303007002002

[5] Clifford SM, Dissanayake C. The early development of joint attention in infants with autistic disorder using home video observations and parental interview. J Autism Dev Disord 2008; 38: 791-805.

http://dx.doi.org/10.1007/s10803-007-0444-7

[6] Zwaigenbaum L, Bryson S, Garon N. Early identification of autism spectrum disorders. Behav Brain Res 2013; 251: 13346.

http://dx.doi.org/10.1016/j.bbr.2013.04.004

[7] Robins DL, Fein D, Barton ML, Green JA. The Modified Checklist for Autism in Toddlers: an initial study investigating the early detection of autism and pervasive developmental disorders. J Autism Dev Disord 2001; 31: 131-144. http://dx.doi.org/10.1023/A:1010738829569

[8] Pierce K, Carter C, Weinfeld M, Desmond J, Hazin R, Bjork $\mathrm{R}$, et al. Detecting, Studying, and Treating Autism Early: The One-Year Well-Baby Check-Up Approach. J Pediatr 2011; 153: 458-465.

http://dx.doi.org/10.1016/j.jpeds.2011.02.036

[9] Rutter M, Bailey A, Lord C. Manual for the Social Communication Questionnaire. Los Angeles: Western Psychological Services 2003.

[10] Wetherby AM, Allen L, Cleary J, Kublin K, Goldstein H. Validity and reliability of the communication and symbolic behavior scales developmental profile with very young children. J Speech Lang Hear Res 2002; 45: 1202-18. http://dx.doi.org/10.1044/1092-4388(2002/097)

[11] Constantino JN, Gruber CP. Social Responsiveness Scale (SRS). Los Angeles, CA: Western Psychological Services 2005.

[12] Dietz $C$, Swinkels $C$, van Daalen $E$, van Engeland $H$, Buitelaar JK. Screening for autistic spectrum disorder in children aged 14-15 months. II: population screening with the early Screening of Autistic Traits Questionnaire (ESAT). Design and general findings. J Autism Dev Disord 2006; 36: 713-22. http://dx.doi.org/10.1007/s10803-006-0114-1

[13] Matson JL, Boisjoli JA, Hess JA, Wilkins J. Factor structure and diagnostic fidelity of the Baby and Infant Screen for Children with aUtlsm Traits-Part 1 (BISCUIT-part 1). Dev Neurorehabil 2010; 13: 72-9. http://dx.doi.org/10.3109/17518420903213576

[14] Gilliam JE. Gilliam Autism Rating Scale, Second Edition (GARS2). Austin, TX: Pro- Ed 2005. 
[15] Nygren G, Sandberg E, Gillstedt F, Ekeroth G, Arvidsson T, Gillberg C. A new screening programme for autism in a general population of Swedish toddlers. Res Dev Disabil 2012; 33: 1200-10.

http://dx.doi.org/10.1016/j.ridd.2012.02.018

[16] Stone WL, Coonrod EE, Ousley OY. Brief report: screening tool for autism in two-year-olds (STAT): development and preliminary data. J Autism Dev Disord 2000; 30(6): 607-12. http://dx.doi.org/10.1023/A:1005647629002

[17] Bryson SE, Zwaigenbaum L, McDermott C, Rombough V, Brian J. The Autism Observation Scale for Infants: scale development and reliability data. J Autism Dev Disord 2007; 38: 731-8. http://dx.doi.org/10.1007/s10803-007-0440-y

[18] Oosterling IJ, Swinkels SH, van der Gaag RJ, Visser JC, Dietz C, Buitelaar JK. Comparative analysis of three screening instruments for autism spectrum disorder in toddlers at high risk. J Autism Dev Disord 2009; 39: 897-909. http://dx.doi.org/10.1007/s10803-009-0692-9

[19] Lord C, Rutter M, DiLavore P, Risi, S. Autism Diagnostic Observation Schedule-WPS Edition. Los Angeles, CA: Western Psychological Services 1999.

[20] Rutter M, Le Couteur A, Lord C. ADI-R. Autism Diagnostic Interview-Revised. WPS Edition. Los Angeles, CA: Western Psychological Services 2003.

[21] Contestabile A, Benfenati F, Gasparini L. Communication breaks-Down: from neurodevelopment defects to cognitive disabilities in Down syndrome. Prog Neurobiol 2010; 91: 122.

http://dx.doi.org/10.1016/j.pneurobio.2010.01.003

[22] Barbaro J, Dissanayake C. Early markers of autism spectrum disorders in infants and toddlers prospectively identified in the Social Attention and Communication Study. Autism. http://www.ncbi.nlm.nih.gov/pubmed/22735682 2013; 17: 6486.

http://dx.doi.org/10.1177/1362361312442597

[23] Osterling J, Dawson G and Munson J. Early recognition of one year old infants with autism spectrum disorder versus mental retardation: a study of first birthday party home videotapes. Dev Psychopathol 2002; 14: 239-51. http://dx.doi.org/10.1017/S0954579402002031

[24] Dewrang $P$, Dahlgren Sandberg A. Parental retrospective assessment of development and behavior in Asperger syndrome during the first 2 years of life. Res Autism Spectr Disord 2010; 4: 461-73.

http://dx.doi.org/10.1016/.rasd.2009.11.003

[25] Mitchell S, Cardy JO, Zwaigenbaum L. Differentiating autism spectrum disorder from other developmental delays in the first two years of life. Dev Disabil Res Rev 2011; 17: 130-40. http://dx.doi.org/10.1002/ddrr.1107

[26] Haglund NG, Källén KB. Risk factors for autism and Asperger syndrome; Perinatal factors and migration. Autism 2011; 15: 163-83. http://dx.doi.org/10.1177/1362361309353614

[27] Norris M, Lecavalier L. Screening accuracy of Level 2 autism spectrum disorder rating scales. A review of selected instruments. Autism 2010; 14: 263-84. http://dx.doi.org/10.1177/1362361309348071

[28] Ehlers S, Gillberg C, Wing L. A screening questionnaire for Asperger syndrome and other high-functioning autism spectrum disorders in school age children. J Autism Dev Disord 1999; 29: 129-41. http://dx.doi.org/10.1023/A:1023040610384

[29] Myles BS, Bock SJ, Simpson RL. Asperger Syndrome Diagnostic Scale. (ASDS). Austin 2001, TX: ProEd.

[30] Gardiner K, Herault Y, Lott IT, Antonarakis SE, Reeves RH, Dierssen M. Down Syndrome: from understanding the neurobiology to therapy. J Neurosci 2010; 30: 14943-5. http://dx.doi.org/10.1523/JNEUROSCI.3728-10.2010

[31] Channell MM, Phillips AB, Loveall SJ, Conners FA, Bussanich PM, Klinger LG. Patterns of autism spectrum symptomatology in individuals with Down syndrome without comorbid autism spectrum disorder. J Neurodev Disord 2015; $7: 5$.

http://dx.doi.org/10.1186/1866-1955-7-5

Received on 21-12-2015

Accepted on 30-12-2015

Published on 07-01-2016

DOI: http://dx.doi.org/10.6000/2292-2598.2015.03.04.9

(C) 2015 Haglund et al.; Licensee Lifescience Global.

This is an open access article licensed under the terms of the Creative Commons Attribution Non-Commercial License (http://creativecommons.org/licenses/by-nc/3.0/) which permits unrestricted, non-commercial use, distribution and reproduction in any medium, provided the work is properly cited. 